\title{
PRODUCTS OF PROTOPOLOGICAL GROUPS
}

\author{
JULIE C. JONES
}

(Received 30 March 2001)

\begin{abstract}
Montgomery and Zippin saied that a group is approximated by Lie groups if every neighborhood of the identity contains an invariant subgroup $H$ such that $G / H$ is topologically isomorphic to a Lie group. Bagley, Wu, and Yang gave a similar definition, which they called a pro-Lie group. Covington extended this concept to a protopological group. Covington showed that protopological groups possess many of the characteristics of topological groups. In particular, Covington showed that in a special case, the product of protopological groups is a protopological group. In this note, we give a characterization theorem for protopological groups and use it to generalize her result about products to the category of all protopological groups.
\end{abstract}

2000 Mathematics Subject Classification. 22A99.

1. Introduction. Montgomery and Zippin [5] saied that a group is approximated by Lie groups if every neighborhood of the identity contains an invariant subgroup $H$ such that $G / H$ is topologically isomorphic to a Lie group. Using a similar idea, Bagley, Wu, and Yang [1] defined a pro-Lie group. Covington [3] extended this concept to topological groups. She defined a protopological group as a group $G$ with a topology $\tau$ and a collection $\mathcal{N}$ of normal subgroups such that (1) for every neighborhood $U$ of the identity, there exists $N \in \mathcal{N}$ such that $N \subseteq U$ and (2) $G / N$ with the quotient topology is a topological group for every $N \in \mathcal{N}$. The collection $\mathcal{N}$ is called a normal system. We denote the quotient topology on $G / N$ by $q_{N}(\tau)$, and we call the collection $2=\left\{q_{N}(\tau)\right\}_{N \in \mathcal{N}}$ a quotient system for $(G, \tau)$. In [2], Covington defines a $t$-protopological group as a protopological group $(G, \tau)$ with the additional requirement that the natural map $\eta_{N}: G \rightarrow G / N$ is an open map for all $N \in \mathcal{N}$. She also shows that the product of $t$-protopological groups is a $t$-protopological group. Although her proof uses ideas different than those used in the proof that a product of topological groups is a topological group, it uses the fact that $\eta_{N}: G \rightarrow G / N$ is an open map for all $N \in \mathcal{N}$. Since there are protopological groups, which are not $t$-protopological, it is of interest to determine if the category of protopological groups is closed under products. However, it is apparent that a different proof technique is needed, since we do not have the hypothesis that $\eta_{N}: G \rightarrow G / N$ is an open map for all $N \in \mathcal{N}$. In this note, we will give a characterization theorem for protopological groups, and then use it to show that the product of protopological groups is protopological.

Let $(G, \tau)$ be a protopological group with normal system $\mathcal{N}$ and quotient system $2=$ $\left\{q_{N}(\tau)\right\}_{N \in \mathcal{N}}$. We note that for each $N \in \mathcal{N}$, the pullback topology from $\left(G / N, q_{N}(\tau)\right)$ determines a group topology on $G$. We will denote this topology on $G$ by $P_{N}(\tau)$. Since the join of group topologies is a group topology, $\tau_{p}=\vee_{N \in \mathcal{N}} P_{N}(\tau)$ is also a group 
topology on $G$. We call $\tau_{p}$ the complete pullback topology on $G$ generated by $\tau$. This topology may also be called the weak topology on $G$. Covington [2] showed that $\tau_{p}$ is the Graev topology when $(G, \tau)$ is a protopological group. It is a well-known result, due to Hewitt and Ross [4], that $\tau_{p}=\vee_{N \in \mathcal{N}} P_{N}(\tau)$ is the coarsest topology that makes $G$ a topological group. Hence, for a protopological group $(G, \tau)$ with normal system $\mathcal{N}$, the complete pullback topology $\tau_{p}$ is the only group topology contained in $\tau$. When using the pullback topologies, we will be interested in saturated sets. In particular, we will say that a set $U \subseteq G$ is saturated with respect to $N \in \mathcal{N}$ if for all $x \in U, \eta_{N}^{-1}\left(\eta_{N}(x)\right) \subseteq U$.

\section{Characterization and product theorems}

THEOREM 2.1 (characterization theorem for protopological groups). Let $(G, \tau)$ be a protopological group with normal system $\mathcal{N}$ and quotient system $2=\left\{q_{N}(\tau)\right\}_{N \in \mathcal{N}}$. Let $\tau^{*}$ be a topology on $G$. Then $\left(G, \tau^{*}\right)$ is a protopological group with normal system $\mathcal{N}$ and quotient system 2 if and only if $\tau^{*}$ satisfies the following properties:

(a) $\tau_{p} \subseteq \tau^{*}$;

(b) if $U \in \tau^{*}$, then $U \in \tau_{p}$ or $U$ is not saturated with respect to $N$ for all $N \in \mathcal{N}$; and

(c) if $U \in \tau^{*}$ is a neighborhood of e, then there exists $N \in \mathcal{N}$ such that $N \subseteq U$.

Proof. Let $\left(G, \tau^{*}\right)$ be a protopological group. Since $\tau_{p}=\vee_{N \in \mathcal{N}} P_{N}(\tau)=\vee_{N \in \mathcal{N}} P_{N}\left(\tau^{*}\right)$ is the coarsest topology that makes $G$ a topological group [4], it follows that $\tau_{p} \subseteq \tau^{*}$. If $U \in \tau^{*}$ is saturated with respect to some $N \in \mathcal{N}$, then $U=\eta_{N}^{-1}\left(\eta_{N}(U)\right)$. But then $U \in$ $P_{N}(\tau) \subseteq \tau_{p}$. Now, if $U \in \tau^{*}$ is a neighborhood of $e$, there exists $N \in \mathcal{N}$ such that $N \subseteq U$. Conversely, assume that (a), (b), and (c) are satisfied. For $N \in \mathcal{N}$, consider the group $G / N$ with the topology $q_{N}\left(\tau^{*}\right)$. Let $U \in q_{N}(\tau)=q_{N}\left(\tau_{p}\right)$. Since $\eta_{N}^{-1}(U) \in \tau_{p} \subseteq \tau^{*}$, it follows that $U \in q_{N}\left(\tau^{*}\right)$. Hence, $q_{N}(\tau)=q_{N}\left(\tau_{p}\right) \subseteq q_{N}\left(\tau^{*}\right)$. Now, let $U \in q_{N}\left(\tau^{*}\right)$. Then $q_{N}^{-1}(U) \in \tau_{p}$ and $q_{N}^{-1}(U)$ is saturated with respect to $N$. Therefore, $q_{N}^{-1}(U) \in \tau_{p}$ which implies that $U \in q_{N}\left(\tau_{p}\right)=q_{N}(\tau)$. Thus, $q_{N}\left(\tau^{*}\right) \subseteq q_{N}\left(\tau_{p}\right)=q_{N}(\tau)$. Therefore, $q_{N}\left(\tau^{*}\right)=q_{N}(\tau)$ for all $N \in \mathcal{N}$.

By imposing the additional condition that $\eta_{N}\left(\tau^{*}\right) \subseteq q_{N}(\tau)$, we obtain a characterization theorem for $t$-protopological groups.

THEOREM 2.2 (product theorem). Let $\left(G_{\alpha}, \tau_{\alpha}\right)$ be a protopological group with normal system $\mathcal{N}_{\alpha}$ and quotient system $\mathscr{2}_{\alpha}=\left\{q_{N}\left(\tau_{\alpha}\right)\right\}_{N \in \mathcal{N}_{\alpha}}$, for all $\alpha \in A$. Let $G=\Pi_{\alpha \in A} G_{\alpha}$, and let $\tau=\Pi_{\alpha \in A} \tau_{\alpha}$ be the product topology on $G$. Then $(G, \tau)$ is a protopological group with normal system $\mathcal{N}=\left\{\prod_{\alpha \in A} N_{\alpha} \mid N_{\alpha} \in \mathcal{N}_{\alpha}\right.$ for all $\alpha \in A$ and $N_{\alpha}=G_{\alpha}$ for all but finitely many $\alpha \in A\}$.

Proof. For each $\alpha \in A$, let $\tau_{p_{\alpha}}$ be the complete pullback topology on $G_{\alpha}$. Then $\left\{\left(G_{\alpha}, \tau_{p_{\alpha}}\right)\right\}_{\alpha \in A}$ is a collection of topological groups, and $\left(G, \tau_{p}\right)$ is a topological group, where $\tau_{p}=\Pi_{\alpha \in A} \tau_{p_{\alpha}}$ is the product topology of $\left\{\tau_{p_{\alpha}}\right\}_{\alpha \in A}$. By the characterization theorem, $\tau_{p_{\alpha}} \subseteq \tau_{\alpha}$ for each $\alpha \in A$. So, $\tau_{p}=\prod_{\alpha \in A} \tau_{p_{\alpha}} \subseteq \Pi_{\alpha \in A} \tau_{\alpha}=\tau$. Now, if $U \in \tau$ is a neighborhood of $e=\left\langle e_{\alpha}\right\rangle_{\alpha \in A} \in G$ then there exists $U_{\alpha_{i}} \in \tau_{\alpha_{i}}$, for $i=1, \ldots, n$, such that $e \in \Pi_{i=1}^{n} U_{\alpha_{i}} \times \Pi_{\alpha \notin\left\{\alpha_{1}, \ldots, \alpha_{n}\right\}} G_{\alpha} \subseteq U$. Then for each $\alpha_{i} \in\left\{\alpha_{1}, \ldots, \alpha_{n}\right\}$, there exists $N_{\alpha_{i}} \in \mathcal{N}_{\alpha_{i}}$ with $N_{\alpha_{i}} \subseteq U_{\alpha_{i}}$. Hence, $N=\Pi_{i=1}^{n} N_{\alpha_{i}} \times \Pi_{\alpha \notin\left\{\alpha_{1}, \ldots, \alpha_{n}\right\}} G_{\alpha} \in \mathcal{N}$ and $e \in N \subseteq$ $\Pi_{i=1}^{n} U_{\alpha_{i}} \times \prod_{\alpha \notin\left\{\alpha_{1}, \ldots, \alpha_{n}\right\}} G_{\alpha} \subseteq U$. Since $\left(G, \tau_{p}\right)$ is a topological group, $\left(G / N, q_{N}\left(\tau_{p}\right)\right)$ is a 
topological group for all $N \in \mathcal{N}$. Hence, $\left(G, \tau_{p}\right)$ is a protopological group with normal system $\mathcal{N}$ and quotient system $2=\left\{q_{N}\left(\tau_{p}\right)\right\}_{N \in \mathcal{N}}$. For each $N \in \mathcal{N}$, let $P_{N}\left(\tau_{p}\right)$ be the pullback topology on $G$ from $\left(G / N, q_{N}\left(\tau_{p}\right)\right)$. Since the complete pullback topology is the only group topology that makes $G$ a protopological group with normal system $\mathcal{N}$ and quotient system 2 , we have that $\tau_{p}=\vee_{N \in \mathcal{N}} P_{N}\left(\tau_{p}\right)$. For $N \in \mathcal{N}$, let $\eta_{N}: G \rightarrow G / N$ be defined by $\eta_{N}(g)=g N$. Now, if $U \in \tau$ is saturated with respect to some $N \in \mathcal{N}$, then, $\eta_{N}(U) \subseteq q_{N}\left(\tau_{p}\right)$. But then, $U=\eta_{N}^{-1}\left(\eta_{N}(U)\right) \in P_{N}\left(\tau_{p}\right) \subseteq \tau_{p}$. So, $U \in \tau_{p}$. By the characterization theorem, $(G, \tau)$ is a protopological group.

\section{REFERENCES}

[1] R. W. Bagley, T. S. Wu, and J. S. Yang, Pro-Lie groups, Trans. Amer. Math. Soc. 287 (1985), no. 2, 829-838. MR 86e:22006. Zbl 0575.22006.

[2] J. L. Covington, T-protopological groups, Topology Proc. 19 (1994), 87-96. MR 96m:54070. Zbl 0847.54036.

[3] _ Protopological groups, Kyungpook Math. J. 35 (1995), no. 2, 323-328. MR 96m:22001. Zbl 0858.22002.

[4] E. Hewitt and K. Ross, Abstract Harmonic Analysis, Springer, New York, 1963.

[5] D. Montgomery and L. Zippin, Topological Transformation Groups, Interscience Publishers, New York, 1955. MR 17,383b. Zbl 0068.01904.

Julie C. Jones: Department of MATHEMATICS, University of Louisiana AT LAFAYetTe, LAFAYETTE, LA 70504, USA

E-mail address: jcj6904@1ouisiana.edu 


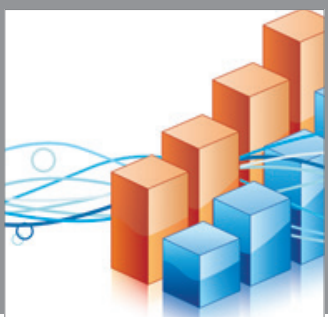

Advances in

Operations Research

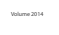

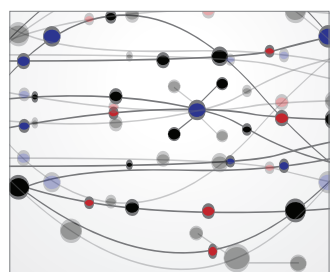

\section{The Scientific} World Journal
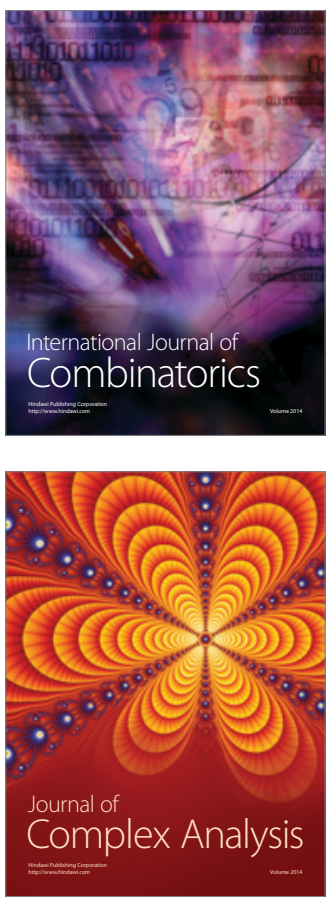

International Journal of

Mathematics and

Mathematical

Sciences
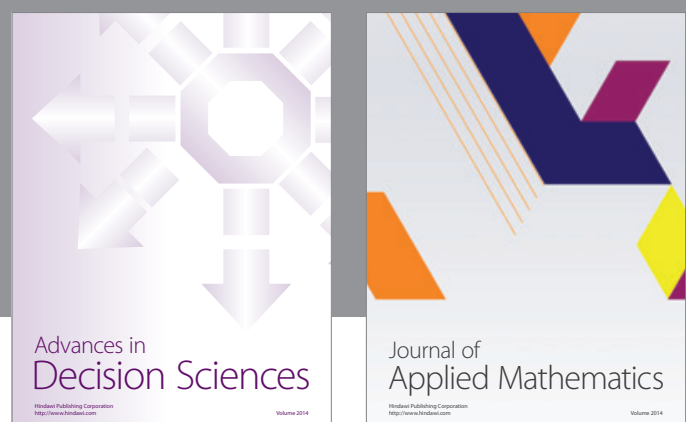

Journal of

Applied Mathematics
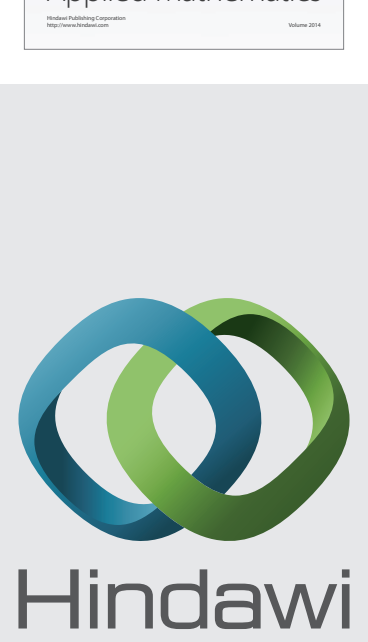

Submit your manuscripts at http://www.hindawi.com
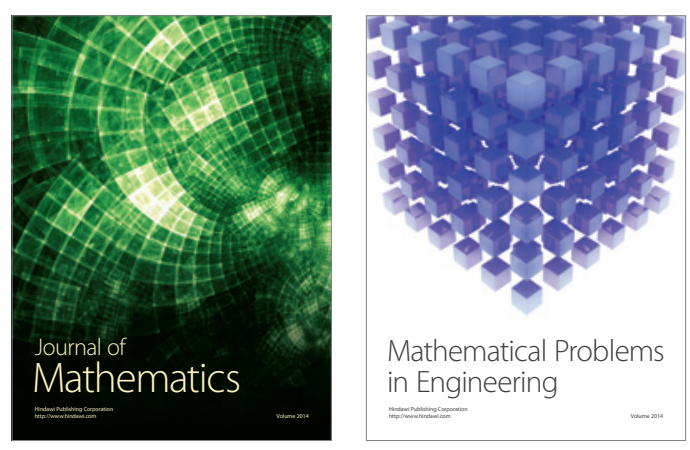

Mathematical Problems in Engineering
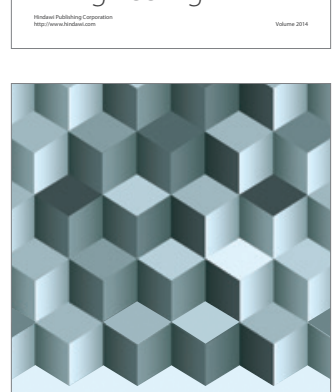

Journal of

Function Spaces
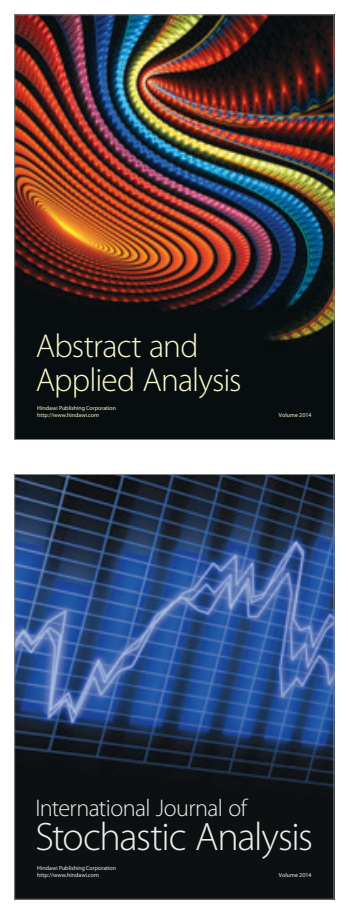

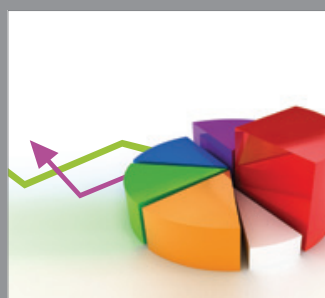

ournal of

Probability and Statistics

Promensencen
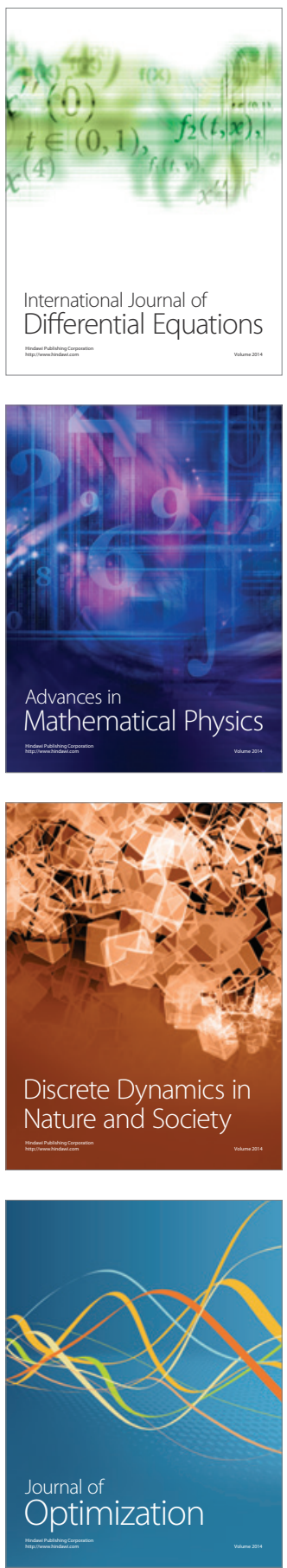